\title{
Beyond artificial intelligence: exploring artificial wisdom
}

\author{
Dilip V. Jeste, ${ }^{1,2,3, *}$ Sarah A. Graham, ${ }^{1,2, *}$ Tanya T. Nguyen, ${ }^{1,2}$ (D) Colin A. Depp, ${ }^{1,2,4}$ \\ Ellen E. Lee, ${ }^{1,2,4, * *}$ and Ho-Cheol Kim ${ }^{5, * *}$ \\ ${ }^{1}$ Department of Psychiatry, University of California San Diego, La folla, CA, USA \\ ${ }^{2}$ Sam and Rose Stein Institute for Research on Aging, University of California San Diego, La folla, CA, USA \\ ${ }^{3}$ Department of Neurosciences, University of California San Diego, La folla, CA, USA \\ ${ }^{4}$ VA San Diego Healthcare System, San Diego, CA, USA \\ ${ }^{5} A I$ and Cognitive Software, IBM Research-Almaden, San fose, CA, USA
}

ABSTRACT

Background: The ultimate goal of artificial intelligence (AI) is to develop technologies that are best able to serve humanity. This will require advancements that go beyond the basic components of general intelligence. The term "intelligence" does not best represent the technological needs of advancing society, because it is "wisdom", rather than intelligence, that is associated with greater well-being, happiness, health, and perhaps even longevity of the individual and the society. Thus, the future need in technology is for artificial wisdom (AW).

\begin{abstract}
Methods: We examine the constructs of human intelligence and human wisdom in terms of their basic components, neurobiology, and relationship to aging, based on published empirical literature. We review the development of AI as inspired and driven by the model of human intelligence, and consider possible governing principles for AW that would enable humans to develop computers which can operationally utilize wise principles and result in wise acts. We review relevant examples of current efforts to develop such wise technologies.
\end{abstract}

Results: AW systems will be based on developmental models of the neurobiology of human wisdom. These AW systems need to be able to a) learn from experience and self-correct; b) exhibit compassionate, unbiased, and ethical behaviors; and c) discern human emotions and help the human users to regulate their emotions and make wise decisions.

Conclusions: A close collaboration among computer scientists, neuroscientists, mental health experts, and ethicists is necessary for developing AW technologies, which will emulate the qualities of wise humans and thus serve the greatest benefit to humanity. Just as human intelligence and AI have helped further the understanding and usefulness of each other, human wisdom and AW can aid in promoting each other's growth

Key words: cognitive activity, aging

\section{Introduction}

The current digital revolution is characterized by a fusion of technologies that are developing at unprecedented rates (Schwab, 2017). This fusion is best exemplified by artificial intelligence (AI), so labeled in 1950 by Turing, who defined AI as the science and engineering of making intelligent machines (Turing, 1950). AI is already commonplace in modern life for accessing information, and is

Correspondence should be addressed to: Dilip V. Jeste, University of California San Diego, 9500 Gilman Drive, Mail Code \#0664, La Jolla, CA, USA. Phone + 1858534 4020. Email: djeste@health.ucsd.edu. Received 21 Feb 2020; revision requested 14 Apr 2020; revised version received 06 May 2020; accepted 09 May 2020. First published online 25 June 2020.

*Co-first authors.

**Co-senior authors. starting to be introduced in healthcare for purposes such as facilitating clinical ordering systems and identifying high-risk patients for screening tests (Reddy et al., 2019). AI will have an increasing impact in healthcare, including psychogeriatrics. The Computing Community Consortium and the Association for the Advancement of Artificial Intelligence recently published a visionary 20 -Year Community Roadmap for AI Research in the US (Gil and Selman, 2019). The ultimate goal here is the development of technology that is best able to serve humanity; this will require advancements that go beyond the basic components of general intelligence.

$\mathrm{AI}$ is superior in some aspects to human intelligence such as visuospatial processing speed and pattern recognition, but lags in terms of reasoning, 
Table 1. Comparison of human intelligence and human wisdom

\begin{tabular}{|c|c|c|}
\hline & HUMAN INTELLIGENCE & HUMAN WISDOM \\
\hline Definition & $\begin{array}{l}\text { A general mental capability that, among other } \\
\text { things, involves the ability to reason, plan, solve } \\
\text { problems, think abstractly, comprehend } \\
\text { complex ideas, learn quickly, and learn from } \\
\text { experience }\end{array}$ & $\begin{array}{l}\text { A uniquely human complex trait including } \\
\text { emotional regulation, pro-social behaviors, } \\
\text { self-reflection, balance between decisiveness } \\
\text { and acceptance of uncertainty, and social } \\
\text { advising }\end{array}$ \\
\hline Assessment & $\begin{array}{l}\text { Standardized tests, e.g. Stanford-Binet IQ test } \\
\text { and WAIS }\end{array}$ & Rating scales, e.g. SD-WISE and 3D-WS \\
\hline Genetics & Strong evidence for heritability & Moderately inherited \\
\hline Neurobiology & $\begin{array}{l}\text { Frontoparietal network for facets like perception, } \\
\text { short-term memory storage, and language }\end{array}$ & $\begin{array}{l}\text { Prefrontal cortex and limbic striatum for } \\
\text { facets like decision-making, problem-solving, } \\
\text { self-control, and emotional regulation }\end{array}$ \\
\hline Modifiability & $\begin{array}{l}\text { Fluid intelligence decreases with age; crystallized } \\
\text { intelligence remains stable until the } 70 \mathrm{~s} \text {. } \\
\text { Intelligence does not increase with aging }\end{array}$ & $\begin{array}{l}\text { Wisdom is adaptive and may increase with age } \\
\text { and personal experience until the } 80 \mathrm{~s} \text {, when it } \\
\text { begins to decline due to cognitive impairment }\end{array}$ \\
\hline $\begin{array}{l}\text { Public health } \\
\text { significance }\end{array}$ & $\begin{array}{l}\text { IQ predicts health and healthcare, educational } \\
\text { achievement, job performance, and income, } \\
\text { but alone is insufficient for the well-being } \\
\text { of the individual or the society }\end{array}$ & $\begin{array}{l}\text { Wisdom is associated with better overall physica } \\
\text { health, mental well-being, happiness, life } \\
\text { satisfaction, resilience, and perhaps longevity; } \\
\text { it is useful for the well-being of the individual } \\
\text { and the society }\end{array}$ \\
\hline
\end{tabular}

new skill learning, and creativity. The current AI is considered narrow or weak, meaning that it is designed to handle only specific tasks. The expected acceleration of technological growth during the next couple of decades will lead to the development of strong or general or broad AI (Kumar and Thakur, 2012). This advanced AI is called artificial general intelligence (AGI), which would be capable of the full range of human cognitive abilities. Beyond AGI, futurists have envisioned Superintelligencean AI that exceeds all human capabilities (Bostrom, 2014).

There is both excitement and concern regarding the super-fast development of AI technologies (Müller and Bostrom, 2016). However, the term "intelligence" does not best represent the technological needs of advancing society, because intelligence alone does not guarantee well-being either for individuals or societies. It is not intelligence, but wisdom, that is associated with greater well-being, happiness, health, and perhaps even longevity of the individual and the society (Jeste et al., 2019). Thus, the future need in technology is for $A W$ and this will also serve to mitigate the risks of advanced AIs such as Superintelligence.

Human wisdom is a scientific construct supported by empirical research during the last 45 years. It has a scientifically based definition, validated measurement scales, underlying neurobiology and genetics, and relationship to aging that are all quite different from those for human intelligence. AW would be modeled after human wisdom and contribute to our understanding of wisdom by clarifying mechanisms or psychological underpinnings. AW development will require close collaboration among computer scientists and engineers, neuroscientists, mental health experts, and ethicists to ensure that AW is developed in a way that best complements and supports human lives. AW will be especially useful for facilitating access to better healthcare even for the most disenfranchised segments of the society such as older mentally ill adults. Below we discuss the constructs of human intelligence, human wisdom, AI, and future AW.

\section{Human intelligence}

\section{Definition}

There is no single agreed-upon definition for intelligence; however, the essence of general intelligence has been defined by experts as "[a] general mental capability that, among other things, involves the ability to reason, plan, solve problems, think abstractly, comprehend complex ideas, learn quickly, and learn from experience" (Gottfredson, 1997, p. 13) (Table 1). Basically, it refers to the ability to acquire and apply knowledge and skills. There have been multiple theories of intelligence, from Spearman's g (general intelligence) factor to Thurstone's primary mental abilities, to Cattell's Gf-Gc (fluid-crystallized) theory and Cattell-Horn-Carroll's theory of cognitive abilities (Goldstein et al., 2015). Although slightly different, most of them agree that intelligence is a multifactorial construct comprised of several components, and integrates a number of cognitive domains and abilities, particularly sensory processing (including attention and working memory), language, acquired knowledge, 
memory consolidation and retrieval, processing and psychomotor speed, and reasoning.

\section{Assessment}

Consistent with the evolution of intelligence theories, intelligence testing has evolved. Today, a number of different standardized intelligence tests exist such as the Stanford-Binet Intelligence Quotient (IQ) test (Terman and Merrill, 1960) and the Wechsler Adult Intelligence Scale Fourth Edition (WAIS IV) (Wechsler, 2008). These tests can reliably measure the various domains associated with intelligence. Many of these tests provide a single IQ score and also subtest or factor scores that map onto and operationalize corresponding theories of intelligence (e.g. four-factor structure of the WAIS: verbal comprehension, perceptual reasoning, processing speed, and working memory).

\section{Genetics}

Intelligence has been historically conceptualized as a highly heritable trait. Studies of twins provide strong evidence for heritability; the intelligence scores of identical twins reared apart are highly correlated (Deary and Penke, 2010).

\section{Neurobiology}

The frontoparietal network is implicated in facets of intelligence including perception, short-term memory storage, and language (Colom et al., 2010).

\section{Modifiability}

Cattell (Cattell, 1963) divided general intelligence into two types: fluid intelligence defined as the ability to use logic and solve problems in new situations without pre-existing knowledge and crystallized intelligence defined as the ability to use previously acquired knowledge. Fluid intelligence decreases with age, beginning in the 20 s or 30 s, but crystallized intelligence remains stable until the 70 s when it may begin to decline. There is no evidence of increase in intelligence with aging. Some specific cognitive skills are malleable to training (Rebok et al., 2014); yet, such interventions do not always generalize across domains (e.g. techniques to improve memory do not help spatial orientation or inductive reasoning) and may not improve overall intellectual functioning (Butler et al., 2018; Sala and Gobet, 2019).

\section{Public health significance}

IQ scores have been shown to predict health and healthcare, educational achievement, job performance, and income (Lloyd, 1978; Spengler et al., 2015); yet, they also have major limitations. People with high
IQs are not necessarily the happiest or most successful (Ali et al., 2013). While traditional intellectual reasoning and procedural knowledge have helped build productive communities, we need emotionally balanced and pro-social frameworks for coping with the uncertainties and complexities of life, and for addressing new challenges of the modern world-e.g. loneliness despite increased digital connections (Lee et al., 2019). Given these considerations, there has been a rising interest in other components of general intelligence. Thus, emotional and social intelligence has been defined as "an array of emotional and social abilities, competencies, and skills that enable individuals to cope with daily demands and be more effective in their personal and social life" (Bar-On et al., 2003; Boyatzis and Gaskin, 2015); however, there are no consensus definitions or commonly used measures for these constructs. At least partly driven by the dissatisfaction with the excessive reliance on intelligence, the field of human wisdom has been attracting increasing attention in recent decades.

\section{Human wisdom}

Wisdom has been discussed in religious and philosophical texts for centuries; however, scientific study of wisdom began in the 1970s. Since then, empirical research on wisdom, long considered the "pinnacle of human condition," has been growing rapidly. The number of peer-reviewed publications on wisdom has increased by several folds during the last couple of decades (Jeste and Lee, 2019).

\section{Definition}

Early wisdom researchers focused on high intelligence as the defining characteristic of human wisdom and stressed that wisdom was a rare quality. However, subsequent investigators emphasized the value of emotions, both in terms of control over negative emotions and of pro-social emotions and behaviors (e.g. empathy and compassion), as being essential for human wisdom. Wisdom is best defined as a uniquely human, complex trait that includes several specific components: emotional regulation (ability to control emotions), pro-social behaviors (e.g. empathy, compassion, and altruism), self-reflection (insight or looking inward), a balance between decisiveness and acceptance of uncertainty and diversity of perspectives, and social advising (Jeste et al., 2019) (Table 1).

\section{Assessment}

A number of rating scales have been developed to measure wisdom such as the Three-Dimensional Wisdom Scale (3D-WS; Thomas et al., 2017). Like other personality traits, wisdom rating scales are based 
on subjective responses to a series of questions. Our group recently published the San Diego Wisdom Scale (SD-WISE; Thomas et al., 2019), which assesses the components of wisdom listed above, and has been shown to be reliable and valid.

\section{Genetics}

Wisdom is a personality trait, i.e. a relatively stable characteristic pattern of a person's thinking, feeling, and behavior. Like resilience and optimism, which are moderately (33-52\%) inherited, wisdom may also be partly inherited and partly influenced by cultural and other environmental factors (Amstadter et al., 2016; Boardman et al., 2008). Wisdom refers to an individual who displays wisdom steadily over time in both their internal processes (e.g. rational thinking, positive emotions) and external activities (e.g. compassionate behavior). At the same time, there need not be a dichotomy between traits and states. The fact that a person's behavior is partly determined by social and other contexts does not negate its being a trait. For example, although intelligence is largely inherited, intelligent people may perform poorly when under duress or in different social contexts. Emotional and behavioral aspects of wisdom are as important as the cognitive ones.

\section{Neurobiology}

The prefrontal cortex (dorsolateral and ventromedial prefrontal cortex as well as anterior cingulate) and limbic striatum (especially amygdala) are critical components of the putative neurocircuitry relevant to wisdom (Meeks and Jeste, 2009). Loss of wisdom without a reduction of IQ has been observed in people with damage to those areas, such as Phineas Gage, as well as in patients with diseases involving these specific brain regions, such as behavioral variants of frontotemporal dementia. There are inevitable overlaps between wisdom and other positive constructs like resilience and social cognition that share certain psychological attributes, including emotional regulation and social decision-making, as well as neurobiological underpinnings such as parts of prefrontal cortex and limbic striatum.

\section{Modifiability}

Wisdom is thought to be adaptive, and unlike intelligence, it can increase with age and personal experience until the 80s when it may begin to decline due to cognitive impairment (Staudinger, 1999). Several components of wisdom have been shown to be present at higher levels in older than in younger adults (Worthy et al., 2011). Additionally, wisdom is more modifiable than intelligence with interventions that can improve emotional regulation and pro-social behaviors (Lee et al., 2020; Treichler et al., 2020).

\section{Public health significance}

A distinct feature of wisdom is its reported usefulness for the well-being of the individual, society, and the species. Wisdom has been associated with positive personal outcomes including better overall physical health, mental well-being, happiness, life satisfaction, resilience, and perhaps longevity (Ardelt, 1997; Bergsma and Ardelt, 2012). Recent studies show that the best correlate of loneliness (a growing global health concern), though in an inverse relationship, is wisdom-i.e. people who score highly in wisdom are significantly less likely to be lonely (Lee et al., 2019). We view loneliness as having cognitive (negative perception of relationships and low self-efficacy), affective (distress due to perceived social isolation), and behavioral (social withdrawal) components. Wisdom can help address all those components through self-reflection (with appropriate perception of relationships and selfefficacy), emotional regulation with positivity (reducing distress, increasing distress tolerance), and greater empathy and compassion toward others as well as self (resulting in greater self-acceptance along with positive social engagement). Thus wisdom, rather than intelligence, may be the antidote for the behavioral toxin of loneliness (Jeste et al., 2020).

\section{Artificial intelligence}

\section{Comparison with human intelligence}

AI systems include components of human intelligence such as reasoning, problem solving, planning, learning, acting, reacting, and understanding and generating language (Gil and Selman, 2019) (Table 2). Some AI systems exceed human intelligence, at least in processing speed and capacity to store information, e.g. GPS apps that provide updated turn-by-turn navigation information in almost any part of the world; the IBM Watson that has defeated Grand Masters of chess and champions of Jeopardy.

\section{Assessment}

Since 1950, the Turing Test has been considered a test of a machine's (computer's) ability to exhibit intelligent behavior equivalent to or indistinguishable from a human's (Turing, 1950). There are ongoing efforts to establish new standards of AI including evaluation metrics (Baudoin et al., 2019).

\section{Benefits and limitations}

A major strength of $\mathrm{AI}$ in healthcare applications is ultra-rapid analysis of large data sets. Today's AI uses rule-based systems to capture high-level articulable patterns and relationships, neural-networkbased deep learning systems to capture low-level, 
Table 2. Current (weak) Al versus future AW: goals and component areas

\begin{tabular}{|c|c|c|}
\hline & CURRENT (NARROW OR SPECIFIC) AI & FUTURE AW \\
\hline Goal & $\begin{array}{l}\text { Accomplish complex human tasks } \\
\text { quickly }\end{array}$ & $\begin{array}{l}\text { Serve as personal assistants to humans to create } \\
\text { a better world }\end{array}$ \\
\hline \multirow{6}{*}{ Component areas } & (1) Comprehension knowledge & (1) Pro-social behaviors (empathy, compassion) \\
\hline & (2) Processing speed & (2) Self-reflection \\
\hline & (3) Quantitative reasoning & (3) Emotional regulation ${ }^{a}$ \\
\hline & (4) Memory & (4) Accepting diversity of perspectives \\
\hline & (5) Visuospatial ability & (5) Decisiveness \\
\hline & (6) Auditory processing & (6) Social advising \\
\hline Assessment & Turing test & Turing-like test for AW \\
\hline \multirow{7}{*}{$\begin{array}{l}\text { Examples of potential } \\
\text { benefits for } \\
\text { healthcare }\end{array}$} & (1) Re-defining diagnoses & (1) Deliver psychotherapy \\
\hline & (2) Biomarker-based subtypes & (2) Facilitate social skill development \\
\hline & (3) Early disease detection & (3) Personal assistant for lifestyle support \\
\hline & (4) Personalized treatment & (4) Companionship to reduce loneliness \\
\hline & (5) Better monitoring & (5) Treatment adherence \\
\hline & (6) Scalable interventions & (6) Real-time assessment of symptoms/emotions \\
\hline & $\begin{array}{l}\text { (7) Efficient operation of systems } \\
\text { (e.g. electronic health records) }\end{array}$ & (7) Facilitate prognosis or treatment expectations \\
\hline
\end{tabular}

${ }^{\mathrm{a} C}$ Computers would not possess this trait but could help a user to improve his/her emotional regulation.

non-articulable patterns and relationships, and also hybrid approaches by combining the two systems (Minsky, 1991). Patterns are useful for making predictions about future health (Graham and Depp, 2019) and efforts are underway to predict disease progression, such as the conversion of mild cognitive impairment (MCI) to Alzheimer's disease, using objective clinical data (Grassi et al., 2018). However, unlike humans, AI cannot yet create a mental model of others' emotions as well as intentions, beliefs, or desires, taking into account social, spatial, temporal, and historical context, and using subjective rather than objective data.

The majority of current AI applications involve making logical decisions or drawing conclusions based on ontology (rules). However, clinical decisionmaking requires more than intelligent thinking - it requires wise thinking that incorporates ethical and moral considerations. Therefore, we are still far from routine adoption of $\mathrm{AI}$ in mental healthcare, especially psychogeriatrics, in view of its limitations and potential risks (Graham et al., 2019, 2020). The incredibly fast advances in computer science and related technologies that have outpaced the development of societal guidelines have raised serious questions about the ethics and morality of AI, and called for international oversight and regulations to ensure safety.

\section{Artificial wisdom}

\section{Human-machine interactions}

Although some futurists have contemplated artificial consciousness (Buttazzo, 2001), it is generally agreed that only humans can have consciousness, autonomy, will, and theory of mind (Leslie, 1987). Thus, only humans can truly be wise as these elements are key to developing/cultivating wisdom. While society/culture shapes many features of wisdom in humans, the self-actualization of humans to achieve these components and develop wisdom is a core element of wisdom. However, humans can develop computers to operationally perform actions that utilize wise principles or result in wise acts (Sevilla, 2013). The key question is whether computers can be designed to adapt and become "wiser" in their algorithms. Unlike humans, computers have the ability to make assessments based on an entire array of data and weigh different inputs with equal or consistent importance every time (no matter what context). Technology can enhance moral, ethical, and pragmatic decision-making through facilitating instantaneous feedback from trusted advisers, or gathering input from, and disseminating data to, large numbers of people at once.

\section{Delivery tools for AI/AW}

Robots are examples of tools delivering AI/AW, interfacing with humans. If we think about consumer-level AW applications, most people will likely have a companion robot (similar to the current ubiquity of smartphones) in the future. Once AW agents are developed, they can be delivered by various other forms (e.g. walls of homes, eye glasses, and hearing aids) to support human well-being. Social robots will be designed to interact with users and proactively communicate with them (for a recent review of behavioral models for social robots, see Nocentini et al., 2019). 


\section{Applying components of human wisdom to computers (AW)}

In the field of robotics, Asimov's three laws are considered central (Asimov, 1950). The first one that supersedes the others is that a robot may not injure a human directly or indirectly. Next in importance is the requirement that a robot must obey human's orders. The last one in the hierarchy is the law that a robot should seek to protect its own existence. Salge and Polani (2017) have suggested replacing Asimov's laws with those based on the principle of robot empowerment in the face of the complex and novel situations that robots will inevitably encounter when interacting with humans. We propose similar rules that would be characteristic of AW systems along with relevant examples of the components of wisdom. Additionally, in Table 2, we provide a comparison of all six main components of human wisdom, with each being followed by a summary of the corresponding characteristics of future AW envisioned.

\section{Three principles of AW systems}

1. A wise system will learn from experience (selfreflection), integrating multiple perspectives (tolerance of diversity/divergent values) as well as past scenarios to make informed decisions and take supported actions.

Thus, AW-based systems will retain and organize memories that are lifelong and life-wide. They will learn from their mistakes and auto-correct to improve future performance (i.e. reinforcement learning) (François-Lavet et al., 2018). Current work (e.g. Qureshi et al., 2018) is focused on developing intrinsically motivated social robots that do not require an external reward system to learn from their mistakes, which will make the implementation of reinforcement learning more suitable for the "reward-sparse" real world.

2. A wise system will exhibit pro-social behaviors (empathy, compassion) in interacting with individuals and model social decision-making (social advising).

For example, AW could serve to facilitate social skill development. A small clinical trial found that in children with autism spectrum disorder, use of wearable technology employing Google Glass, which encourages facial engagement and provides cues about the emotions of their social partners during interactions, was associated with significant improvements in socialization skills (Voss et al., 2019).

3. A wise system will be able to discern human emotions and help people to emotionally regulate and exercise good judgment or knowledge (act decisively).

Thus, AW can help promote emotional regulation in its users. A number of randomized controlled trials (RCTs) have shown that emotional regulation can be enhanced (Lee et al., 2020). A wise system could act as a "wisdom coach" to provide a cognitive reappraisal of a stressful situation through appropriate role modeling and thus help the user to reinterpret the meaning of a distressing event and control negative emotions. For example, Hao et al. (2019) used a robot to regulate a user's emotional state from negative to positive emotions, or to maintain positive emotions, during a simulated "waiting" scenario (as in waiting in line, to be seated, etc.).

\section{Assessment: Turing-like test for AW}

Based on the principles of the original Turing test for AI, we propose that the performance metrics for AW will involve a human evaluator who will compare her or his natural language conversations with a human versus those with an AW system in response to specific questions for assessing wisdom. The questions may come from moral dilemmas (e.g. the Trolley Problem) commonly used in the literature (Christensen et al., 2014; Thomson, 1976). The evaluator will be blinded to which of the two partners in conversation is a machine. The conversation will be limited to a text-only channel, such as a computer keyboard and screen, so the result will not depend solely on the machine's ability to render words as speech. If the evaluator cannot reliably discern the AW system from the human, the AW system will be said to have passed the AW test.

\section{Public health benefit}

In a rapidly changing world, unexpected global crises may dramatically change the traditional norms of behavior in a very short time. How will machines handle these challenges that tax even human wisdom in unforeseen ways? This type of situation is exemplified by the novel coronavirus pandemic, which has sowed high levels of uncertainty, confusion, and emotional unease, given that humans had no previous exposure to this virus. Another challenge has resulted from the recommended steps to control the spread of the virus, through social distancing, which reduces social engagement, one of the most evidence-based strategies for health and longevity. There are also individual-level moral dilemmas for healthcare workers and others to weigh personal health risks against their work responsibilities and compassion toward others, and for allocation of scarce resources among patients. A wise system would need to promote personalized wisdom for different users under different conditions and at different times.

\section{Conclusions}

If we conceptualize future AI technologies as AW, we can ensure that these technologies are designed 
to emulate the qualities of wise humans and thus serve the greatest benefit to humanity. In the area of psychogeriatrics, AI/AW will increasingly be a part of digital medicine and will contribute to mental health research and practice. As researchers and practitioners vested in improving mental healthcare, we must take an active role in informing the introduction of AI/AW into clinical care by lending our clinical expertise and collaborating with data and computational scientists, as well as other experts, to help transform mental health practice and improve care for patients.

Intelligence is necessary but not sufficient for wisdom. Intelligence is needed for the survival of Homo sapiens, but wisdom is critical for thriving in the modern society. Wisdom is potentially more modifiable than IQ, and unlike IQ, it may increase with age and experience. AI technologies of the future will require new conceptual and computational models based on human wisdom and not human intelligence, to produce AW. An AW agent can serve as an assistant, peer, or coach. It can help make humans wiser. A long-term transdisciplinary collaboration is essential for the development of AW, which will have far greater positive impact on human well-being than any technology developed to date.

\section{Source of funding}

This work was supported, in part, by the National Institute of Mental Health [T32 Geriatric Mental Health Program (grant MH019934 to DVJ [PI]), an R01 grant (R01MH094151-01 to DVJ [PI]), a K23 grant (K23MH118435-01A1 to TTN [PI]), a K23 grant (K23MH119375-01 to EEL [PI])], the Sam and Rose Stein Institute for Research on Aging, University of California San Diego, the NARSAD Young Investigator grant from the Brain and Behavior Research Foundation (PI: Ellen E. Lee, $\mathrm{MD}$ ), and by the IBM Research AI through the AI Horizons Network.

\section{References}

Ali, A. et al. (2013). The relationship between happiness and intelligent quotient: the contribution of socio-economic and clinical factors. Psychological Medicine, 43(6), 1303-1312. doi: 10.1017/S0033291712002139 PMID: 22998852.

Amstadter, A. B., Moscati, A., Oxon, M. A., Maes, H. H., Myers, J. M. and Kendler, K. S. (2016). Personality, cognitive/psychological traits and psychiatric resilience: a multivariate twin study. Personality and Individual Differences, 91, 74-79. doi: 10.1016/j.paid.2015.11.041 PMID: 29104336.
Ardelt, M. (1997). Wisdom and life satisfaction in old age. The fournals of Gerontology Series B: Psychological Sciences and Social Sciences, 52(1), P15-P27. doi: 10.1093/geronb/52b .1.p15 PMID: 9008672.

Asimov, I. (1950). Runaround. In: I, Robot (p. 40). New York: Doubleday.

Bar-On, R., Tranel, D., Denburg, N. L. and Bechara, A. (2003). Exploring the neurological substrate of emotional and social intelligence. Brain, 126(8), 1790-1800. doi: 10.1093/brain/awg177 PMID: 12805102.

Baudoin, C. R., Johnson, L. L. and Rivett, P. (2019). Artificial Intelligence and OMG Standards. Object Management Group. Available at: https://www.nist.gov/ system/files/documents/2019/06/10/nist-ai-rfi-omg001.pdf

Bergsma, A. and Ardelt, M. (2012). Self-reported wisdom and happiness: an empirical investigation. Fournal of Happiness Studies, 13(3), 481-499. doi: 10.1007/s 10902-011-9275-5.

Boardman, J. D., Blalock, C. L. and Button, T. M. (2008). Sex differences in the heritability of resilience. Twin Research and Human Genetics: The Official fournal of the International Society for Twin Studies, 11(1), 12-27. doi: 10.1375/twin.11.1.12 PMID: 18251671.

Bostrom, N. (2014). Superintelligence: Paths, Dangers, Strategies. Oxford: Oxford University Press. doi: 10.5860/ choice. 187536.

Boyatzis, R. E., Gaskin, J. and Wei, H. (2015). Emotional and social intelligence and behavior. In: S. Goldstein, D. Princiotta and J. Naglieri (Eds.), Handbook of Intelligence (pp. 243-262). New York: Springer. doi: 10.1007/978-14939-1562-0_17.

Butler, M. et al. (2018). Does cognitive training prevent cognitive decline? A systematic review. Annals of Internal Medicine, 168(1), 63-68. doi: 10.7326/M17-1531 PMID: 29255842.

Buttazzo, G. (2001). Artificial consciousness: utopia or real possibility? Computer, 34(7), 24-30. doi: 10.1109/2 .933500 .

Cattell, R. B. (1963). Theory of fluid and crystallized intelligence: a critical experiment. Fournal of Educational Psychology, 54, 1-22. doi: 10.1037/h0046743.

Christensen, J. F., Flexas, A., Calabrese, M., Gut, N. K. and Gomila, A. (2014). Moral judgment reloaded: a moral dilemma validation study. Frontiers in Psychology, 5, 607. doi: 10.3389/fpsyg.2014.00607 PMID: 25071621.

Colom, R., Karama, S., Jung, R. E. and Haier, R. J. (2010). Human intelligence and brain networks. Dialogues in Clinical Neuroscience, 12(4), 489. PMID: 21319494.

Deary, I. J., Penke, L. and Johnson, W. (2010). The neuroscience of human intelligence differences. Nature Reviews Neuroscience, 11(3), 201-211. doi: 10.1038/ nrn2793 PMID: 20145623.

François-Lavet, V., Henderson, P., Islam, R., Bellemare, M. G. and Pineau, J. (2018). An introduction to deep reinforcement learning. Foundations and Trends ${ }^{\circledR}$ in Machine Learning, 11(3-4), 219-354. doi: 10.1561/ 2200000071.

Gil, Y. and Selman, B. (2019). A 20-Year Community Roadmap for Artificial Intelligence Research in the US. Computing Community Consortium (CCC) and Association for the Advancement of Artificial Intelligence 
(AAAI). August 6. Available at: https://cra.org/ccc/ resources/workshopreports/

Goldstein, S., Princiotta, D. and Naglieri, J. A. (2015). Handbook of Intelligence: Evolutionary Theory, Historical Perspective, and Current Concepts. New York: Springer. doi: 10.1007/978-1-4939-1562-0.

Gottfredson, L. S. (1997). Mainstream Science on Intelligence (editorial). Intelligence, 24(1), 13-23. doi: 10 .1016/s0160-2896(97)90011-8.

Graham, S. A. and Depp, C. A. (2019). Artificial intelligence and risk prediction in geriatric mental health: what happens next? International Psychogeriatrics, 31(7), 921-923. doi: 10.1017/S1041610219000954.

Graham, S. A. et al. (2019). Artificial intelligence for mental health and mental illnesses: an overview. Current Psychiatry Reports, 21(11), 116. doi: 10.1007/s11920-019-1094-0 PMID: 31701320.

Graham, S. A. et al. (2020). Artificial intelligence approaches to predicting and detecting cognitive decline in older adults: a conceptual review. Psychiatry Research, 284, 112732. doi: 10.1016/j.psychres.2019.112732 PMID: 31978628.

Grassi, M., Loewenstein, D. A., Caldirola, D., Schruers, K., Duara, R. and Perna, G. (2018). A clinically-translatable machine learning algorithm for the prediction of Alzheimer's disease conversion: further evidence of its accuracy via a transfer learning approach. International Psychogeriatrics, 31(7), 1-9. doi: 10.1017/ S1041610218001618 PMID: 30426918.

Hao, M., Cao, W., Liu, Z., Wu, M. and Yuan, Y. (2019). Emotion regulation based on multi-objective weighted reinforcement learning for human-robot interaction. In: 2019 12th Asian Control Conference (ASCC), June 9 (pp. 1402-1406). IEEE. Available at: https://ieeexplore.ieee .org/abstract/document/8764941.

Jeste, D. V. et al. (2019). The new science of practical wisdom. Perspectives in Biology and Medicine, 62(2), 216-236. doi: 10.1353/pbm.2019.0011 PMID: 31281119.

Jeste, D. V. and Lee, E. E. (2019). Emerging empirical science of wisdom: definition, measurement, neurobiology, longevity, and interventions. Harvard Review of Psychiatry, 27, 127-140. doi: 10.1097/HRP.0000000000000205 PMID: 31082991.

Jeste, D. V., Lee, E. E. and Cacioppo, S. (2020). Battling the modern behavioral epidemic of loneliness: Suggestions for research and interventions. $\mathcal{F} A M A$ Psychiatry, 77(6), 553-554. doi: 10.1001/jamapsychiatry.2020.0027.

Kumar, K. and Thakur, G. S. (2012). Advanced applications of neural networks and artificial intelligence: a review. International fournal of Information Technology and Computer Science, 4(6), 57. doi: 10.5815/ijitcs.2012.06.08.

Lee, E. E. et al. (2019). High prevalence and adverse health effects of loneliness in community-dwelling adults across the lifespan: role of wisdom as a protective factor. International Psychogeriatrics, 31, 1447-1462. doi: 10.1017/S104161021 8002120 PMID: 30560747.

Lee, E. E. et al. (2020). Outcomes of randomized clinical trials of interventions to enhance social, emotional, and spiritual components of wisdom: a systematic review and meta-analysis. FAMA Psychiatry. doi: 10.1001/ jamapsychiatry.2020.0821.
Leslie, A. M. (1987). Pretense and representation: the origins of "theory of mind". Psychological Review, 94(4), 412. doi: 10.1037/0033-295X.94.4.412.

Lloyd, D. N. (1978). Prediction of school failure from third-grade data. Educational and Psychological Measurement, 38(4), 1193-1200. doi: 10.1177/ 001316447803800442.

Meeks, T. W. and Jeste, D. V. (2009). Neurobiology of wisdom: an overview. Archives of General Psychiatry, 66, 355-365. doi: 10.1001/archgenpsychiatry.2009.8 PMID: 19349305.

Minsky, M. L. (1991). Logical versus analogical or symbolic versus connectionist or neat versus scruffy. AI Magazine, 12(2), 34. doi: 10.1609/aimag.v12i2.894.

Müller, V. C. and Bostrom, N. (2016). Future progress in artificial intelligence: a survey of expert opinion. In: Fundamental Issues of Artificial Intelligence (pp. 555-572). Cham: Springer.

Nocentini, O., Fiorini, L., Acerbi, G., Sorrentino, A., Mancioppi, G. and Cavallo, F. (2019). A survey of behavioral models for social robots. Robotics, 8(3), 54. doi: $10.3390 /$ robotics 8030054 .

Qureshi, A. H., Nakamura, Y., Yoshikawa, Y., and Ishiguro, H. (2018). Intrinsically motivated reinforcement learning for human-robot interaction in the real-world. Neural Networks, 107, 23-33. doi: 10.1016/j.neunet.2018.03 .014 PMID: 29631753.

Rebok, G. W. et al. (2014). Ten-year effects of the advanced cognitive training for independent and vital elderly cognitive training trial on cognition and everyday functioning in older adults. Fournal of the American Geriatrics Society, 62(1), 16-24. doi: 10.1111/jgs.12607 PMID: 24417410.

Reddy, S., Fox, J. and Purohit, M. P. (2019). Artificial intelligence-enabled healthcare delivery. Fournal of the Royal Society of Medicine, 112(1), 22-28. doi: 10.1177/ 0141076818815510 PMID: 30507284.

Sala, G. and Gobet, F. (2019). Cognitive training does not enhance general cognition. Trends in Cognitive Sciences, 23(1), 9-20. doi: 10.1016/j.tics.2018.10.004 PMID: 30471868.

Salge, C. and Polani, D. (2017). Empowerment as replacement for the three laws of robotics. Frontiers in Robotics and AI, 4, 25. doi: 10.3389/frobt.2017.00025.

Schwab, K. (2017). The Fourth Industrial Revolution. New York: Currency.

Sevilla, D. C. (2013). The quest for artificial wisdom. AI E Society, 28, 199-207. doi: 10.1007/s00146-0120390-60.

Spengler, M., Brunner, M., Damian, R. I., Lüdtke, O., Martin, R. and Roberts, B. W. (2015). Student characteristics and behaviors at age 12 predict occupational success 40 years later over and above childhood IQ and parental socioeconomic status. Developmental Psychology, 51(9), 1329. doi: 10.1037/ dev0000025 PMID: 26147775.

Staudinger, U. M. (1999). Older and wiser? Integrating results on the relationship between age and wisdom-related performance. International fournal of Behavioral Development, 23(3), 641-664. doi: 10.1080/ 016502599383739 . 
Terman, L. M. and Merrill, M. A. (1960). Stanford-Binet Intelligence Scale: Manual for the Third Revision, Form $L-M$. Boston, MA: Houghton Mifflin.

Thomas, M. L. et al. (2019). A new scale for assessing wisdom based on common domains and a neurobiological model: the San Diego Wisdom Scale (SD-WISE). Fournal of Psychiatric Research, 108, 40-47. doi: 10.1016/j.jpsychires .2017.09.005 PMID: 28935171.

Thomas, M. L., Bangen, K. J., Ardelt, M. and Jeste, D. V. (2017). Development of a 12-item abbreviated threedimensional Wisdom Scale (3D-WS-12): item selection and psychometric properties. Assessment, 24, 71-82. doi: 10.1177/1073191115595714 PMID: 26209513.

Thomson, J. J. (1976). Killing, letting die, and the trolley problem. Monist, 59, 204-217. doi: 10.5840/ monist197659224 PMID: 11662247.

Treichler, E. B. et al. (2020). A pragmatic trial of a group intervention in senior housing communities to increase resilience. International Psychogeriatrics, 32(2), 173-182. doi: 10.1017/S1041610219002096.

Turing, A. M. (1950). Computing machinery and intelligence. Mind, 49, 433-460.

Voss, C. et al. (2019). Effect of wearable digital intervention for improving socialization in children with autism spectrum disorder: A randomized clinical trial. $\mathcal{F} A M A$ Pediatrics, 173(5), 446-454. doi: 10.1001/jamapediatrics.2019.0285 PMID: 30907929.

Wechsler, D. (2008). Wechsler Adult Intelligence Scale Fourth Edition: Technical Interpretive Manual. San Antonio, TX: NCS Pearson.

Worthy, D. A., Gorlick, M. A., Pacheco, J. L., Schnyer, D. M. and Maddox, W. T. (2011). With age comes wisdom: decision making in younger and older adults. Psychological Science, 22(11), 1375-1380. doi: 10.1177/0956797611420301 PMID: 21960248. 